\title{
Medical Service Platform and Internet+ Medicine Innovation Construction
}

\author{
Xiaoqing Feng, Yanan Cao and Yangyang Li \\ Department of Information Engineering, Heilongjiang International University, \\ Harbin, China \\ 2500425970@qq.com
}

\begin{abstract}
Internet+ medicine is experiencing an information age with rapid development. Medical institutions are setting up the network information system to offer a convenient medical service platform to patients and ordinary people, which is beneficial to people and can solve the many medical problems that have long been brought to patients. This paper studies the Internet+ medical service platform. A new complete scientific safe medical platform system is established by improving the safety of the information system, formulating a relevant management system, formulating relational laws and regulations, increasing policy support and supervision, and developing awareness of protecting personal information.
\end{abstract}

Keywords: Internet+, Innovative medical care, Medical reform, Medical service platform, Big data analysis

\section{Development environment and advantages of Internet+ medicine}

At present, most Internet+ industries depend on support from national policies to create products for profit. In this epoch when internet+ medicine is popular, Internet+ medical applications are becoming more and more. According to incomplete statistics, the types have been more than 2,000. However, there is still no product that can solve major medical problems effectively nowadays [1][2].

With the continuous development of society, people attach more attention to physical health, so the demand for the medical industry is stricter. The traditional way cannot satisfy people anymore. And the key solution to the problem is innovation, which also provides an open market to the Internet+ medicine industry.

First of all, Internet+ medicine can solve some problems which traditional medicine has nothing to do with. For example, the medical information between doctors and patients cannot be updated timely and patients have no idea to realize experts' information, so patients cannot choose the proper doctors so the treatments are delayed. "Difficult to see a doctor, expensive to see a doctor" has become one of the major problems in society [3].

Then, Internet+ medicine can better allocate medical resources and reduce resource waste. From the perspective of patients, they can choose to seek medical treatment online when they have common diseases, which can not only save medical expenses, but also save time, and also breaks the limitation of space and time. From the perspective of doctors, Internet+

Article history:

Received (January 10, 2019), Review Result (February 10, 2019), Accepted (March 14, 2019) 
medicine provides much more convenience. Doctor clinical cases can be spread quickly without the limitation of time and space, so the fame of the doctor will improve. It provides more choices for patients and also increases the income of doctors [4].

Last is the safety problem. With the continuous completion of the internet, people pay more and more attention to internet safety. In the era of mobile internet, Internet+ medicine based on APP also faces security threats. As for the development of the whole industry, the network security problem must be solved, otherwise, the real function of Internet+ medicine cannot be realized [5].

\section{Internet+ medicine development trend}

Now there is an urgent problem that it is difficult for people to see a doctor. To solve this problem, Internet+ medicine is launched to simplify the treatment process through the Internet and optimize the medical treatment process. Internet+ medicine will be high-profile in the future, and medical models related to Internet+ medicine will spring up continuously. Internet+ medicine will cover every aspect of the medical industry. In the end, people will realize online appointment registration, get medical advice online, remote consultations, and buy medicine online.

Medical quality and medical accuracy are the two main issues that concern Internet+ medicine. Some western countries have begun to study medical precision. Medical precision is mainly reflected in the following two points: precise visits and precise treatment. We can ensure accurate treatment only when we solve the problem of accurate information between doctors and patients.

Internet+ medicine cannot flourish without support from government policy. In the context of open policy, medicine has achieved great success, and continuous innovation and development also promote further institutional reforms in the medical field. On the base of the internet, the relationship between doctors and patients develops in harmony. The best edge is the help of rational allocation of resources.

At present, the information on the medicine industry is relatively isolated. It cannot be shared and exchanged timely, so the trend of an information barrier is formed, which impedes the further implementation of policies. Therefore, solving this problem with Internet+ medicine is urgent. Creating a healthy and safe information system to break the information barrier is the main mission of Internet+ medicine development.

\section{Internet+ medicine problems and analysis}

\subsection{The particularity of the industry}

Internet+ medicine development is limited by the particularity of the industry. This particularity is manifested in the way that patients are more willing to choose face-to-face treatments and are more likely to accept the traditional medical treatment process psychologically. Although Internet+ medicine provides a convenient medical platform online, the sense of distance in the medical treatment process will always make patients lose confidence in the doctors and cause many concerns about the medical treatment, and the accuracy of diagnosis. 


\subsection{Barriers to medical data}

The premise of Internet+ medicine development is the benign development of the medical industry. The character of this model is using the Internet to integrate the medical system deeply to maximize the medical effects. Medical data plays an important role in the Internet+ medicine model. However, patient's clinical information is personal privacy legally, which leads to the inability to share medical data in all directions, limiting the construction of Internet+ medicine mode and the promotion of reform

\subsection{Immature business model}

Internet+ medicine has derived two models. One is online consultation tools, including medical websites, BBS platforms, and real-time interactive modules. But this mode is not accepted by most patients because of the business element, and it cannot realize the purpose of publicity of medical units. The other is medical devices that can access the network. The concept of this mode is good, it can detect various physical signs of patients timely and provide parameters for personalized treatment. But the implementation needs considerable financial support, which cannot be spread among general patients, so it limits the further development of Internet+ medicine.

\section{Recommendations for Internet+ medical development}

Under the background of deepening the medicine reform and advocating the concept of deep integration of Internet+ in every industry, entrepreneurs have gained many opportunities to make innovation and entrepreneurship, but both industry and entrepreneurs are under big pressure simultaneously. In the construction process of the Internet+ medicine platform, firstly, we should conform to the advantages of traditional medicine, and then retain the essence and discard the dross to refine networked information in traditional medical service system, to guide Internet+ medicine platform. Next, we should adopt the "online + offline" combined medical model to enhance the transparency of drug prices, optimize the treatment process, and solve medical problems such as "drugs are expensive, seeing the doctor is difficult".

\section{5. "Internet+ medical health" should develop within innovation}

Recently the State Council issued the Opinions on Promoting the Development of "Internet+ Medical Health, mentioning the development strategy of deep integration of the Internet and medical health. In the context of the Internet, medicine reform continues to deepen. At the same time, the opinion promoted the implementation of the National Health Policy. The opinion mainly focused on the people's difficulty in seeing a doctor. It also proposed guidelines for improving people's health by taking medicine reform as an opportunity. From April 12th when the State Council executive meeting determined to develop "Internet+ medical health" measures to April 16th when the State Council Information Office published relative policies, people in the different areas were full of expectations on this opinion. At the same time, people in a different areas interpreted the core connotation of the Opinion and integrate the inherent resources of every area, to service the Internet+ medicine platform. The publication of the opinion received a warm response from the media and markets and is regarded as one of the most pragmatic documents in the field of medical reform in recent years. 
The Opinion defined the guidance of Internet+ medicine in policy, and the government espoused and encouraged the enthusiasm of the innovation team. It also confirmed the emphasis on Internet+ medicine and expanded understanding of Internet+ medicine. It no longer depended only on the internet to seek doctors or medicine but expanded comprehensively on the pragmatic application of the medical system, such as family doctors, smart medicine, drug supply, etc. The Opinion also defined the behavioral boundary of "Internet+ Medical Health" and proposed rectification opinions on the three most controversial issues in practice, including online medical consultation, online hospital, and prescription drug e-commerce. The opinion confirmed that the online hospital must be based on the entity hospital, and confirmed responsibility attribution of internet medical platforms for third-party organizations. Responsibility attribution is one of the necessary conditions of the Internet+ medicine system because internet medicine is related to people's life security. Therefore, third-party organizations should ensure that the qualifications of service personnel comply with relevant regulations, and that service personnel should shoulder corresponding responsibilities.

All in all, the publication of the Opinion is the response to current social demands. It is also a summary and phased improvement of experience from previous medical institution platforms and regional governments about Internet+ medicine. In the future, we should under the premise of rigorous and inclusive, espouse and encourage the innovative development of Internet+ medicine continuously, with an unremitting attitude to innovate and reform, to promote the implementation of the healthy China strategy.

\section{Security problem}

\subsection{Medical information}

The continuous completion of Internet+ promotes the sound development of all areas. The internet theoretically follows the OSI/RM structure which is the open system interconnection reference model. What the entire network system emphasizes is not only the massive storage and transmission of data but even the open and interconnected working mode. The use of the mode will put the network under hacking, spoofing, virus propagation, etc., so we should pay attention to information security and take protective measures when we construct the Internet+ medicine platform. In Internet+ medicine, patients' relevant private information may be disclosed when they make online appointments, have online consultations, and purchase medicines online. In addition, there are certain risks during the payment of treatment and medicine. These sensitive data are very likely to be defrauded and stolen by criminals, threatening the vital interests of patients. The patient's medical record data and test results will have a serious impact if they are leaked. Therefore, the database should be kept safe to prevent leakage and cause unnecessary losses.

\subsection{Hospital information system}

Another major problem the "Internet+" faces is the security of the hospital information system. How to guarantee the security of the information is one of the first considerations for the reformists in the whole industry. In the traditional hospital system, information is isolated and closed. Although the process of collecting data is cumbersome and affects the efficiency of hospital work, this information is rarely leaked with its physical security features, compared with the data exposed on the Internet. At present, traditional hospital information management systems cannot defend against threats, and there is no distinct security division 
inside the network, so the security protection of the border is not tight enough and there are many security issues. Once these problems are discovered by network intermediaries, they may cause information leakage, resulting in unimaginable losses.

\section{Countermeasures}

\subsection{Enhance information system security}

To ensure the security of the data in the system, hospitals and other medical institutions should invest a large amount of manpower, material resources, and financial resources to realize the concentration of human resources and the standardization of information security departments gradually. The database of the hospital can rely on personalized customization from a professional software development company to make sure the management system is complete and safe. In addition, to prevent private data leakage in hospitals and medical institutions, data can be encrypted and make advanced coding, and also set dedicated backup system for hospitals and medical institutions, to reduce the BUG in the information management system and minimize insecurity.

\subsection{Establish a corresponding management system}

\subsubsection{Rights assignment}

Manage and control some inner data of medical institutions by the strategy of rights assignment. Effectively separate the access rights of individuals and organizations, and limit the scope and number of accesses. Large medical departments can ensure data security by the way of the cluster. When the data is disclosed, the reason and person in charge can be found in time, so that the hospital can investigate relevant legal departments and personnel.

\subsubsection{Staff training}

Medical institutions should conduct training in safety awareness and safety skills for database administrators and operators. It will not only be beneficial to improve the professional responsibility of the staff and improve safety awareness, but also improve management efficiency from a technical perspective.

\subsection{Formulating relevant laws and regulations}

The state should study and introduce management measures to regulate Internet diagnosis and treatment as soon as possible. Government should improve policy support and supervision, and strengthen supervision and service levels continuously. The localized management program implements the goal of unified supervision online and offline. Management institutions should also establish a supervision port for the medicine administration. All medical institutions with internet medical service and all internet medicine platforms should push, back up, and update data to the regional comprehensive health information platform timely, and medicine administration departments will supervise internet medical behaviors dynamically through a supervision port. At the same time, the government can increase policy support, and grant conditions like tax incentives to enterprises that develop Internet medical databases and enterprises that provide Internet medical services, to promote the rapid development and progress of the Internet medical industry. 


\subsection{Develop awareness of protecting personal information}

Patients should develop an awareness of protecting personal information. It is necessary to improve awareness of security continuously, learn security knowledge, change passwords regularly, and increase password difficulty by reading the hospital and related documents in government and society. Don't log in or visit unidentified websites. Clear the browsing records and traces after surfing the Internet in the public network, leaving no opportunity for others to threaten personal information security.

\section{Conclusions}

The information age is developing rapidly, and Internet+ has caused widespread concern in society. The state continuously increases the support and encouragement of the Internet+ industry. The reform of the Internet+ industry has officially kicked off, and the Internet+ medical industry is even more imposing. However, when the Internet+ and medicine combine deeply, we should study problems faced by the combination from different aspects. In the development, we must not only carry out industrial innovation but also discover and solve some problems in time, so that the new industry will gradually mature after continuous improvement. In the future, Internet+ medicine will develop continuously, waiting for the opportunity and facing the challenge.

\section{Acknowledgments}

This work was supported by Heilongjiang University College Students Innovation and Entrepreneurship Training Program (201813296010)

\section{References}

[1] $\mathrm{Xu} \mathrm{Mu,} \mathrm{"Lack} \mathrm{of} \mathrm{attention} \mathrm{to} \mathrm{system} \mathrm{security} \mathrm{protection.} \mathrm{Many} \mathrm{foreign} \mathrm{medical} \mathrm{institutions} \mathrm{were} \mathrm{attacked} \mathrm{by}$ blackmail software," Information Security and Communication Confidentiality, pp.5-10, (2016)

[2] Daren Zhao, Sichang He, Boxing Sun, Zhihui Liu, and Ruihua Zhang, "Implementation status and thinking of Internet+ medical care in China," Health Economics Research, pp.7-16, (2017)

[3] Xiaofeng Zhang and Hui Lin. "Research on Internet health-related online health service industry," Sichuan Medical, vol.6, pp.12-19, (2016)

[4] Youhong Li, "Computer network security technology and protection strategy," Electronic Testing, vol.9, no.9, pp.42-49, (2014)

[5] Anqi Wang, et al., "Current situation of internet medical operation in China: Based on the investigation and analysis of three hospitals," Research on China's Health Policy, pp.12-18, (2016) 